Delft University of Technology

\title{
Wafer-Scale Graphene-Based Soft Electrode Array with Optogenetic Compatibility
}

Velea, A.I.; Vollebregt, S.; Wardhana, G.K.; Giagka, V.

DOI

10.1109/MEMS46641.2020.9056367

Publication date

2020

Document Version

Accepted author manuscript

Published in

33rd IEEE International Conference on Micro Electro Mechanical Systems, MEMS 2020

\section{Citation (APA)}

Velea, A. I., Vollebregt, S., Wardhana, G. K., \& Giagka, V. (2020). Wafer-Scale Graphene-Based Soft Electrode Array with Optogenetic Compatibility. In 33rd IEEE International Conference on Micro Electro Mechanical Systems, MEMS 2020 (pp. 421-424). [9056367] (Proceedings of the IEEE International Conference on Micro Electro Mechanical Systems (MEMS); Vol. 2020-January). IEEE Sensors. https://doi.org/10.1109/MEMS46641.2020.9056367

Important note

To cite this publication, please use the final published version (if applicable).

Please check the document version above.

\section{Copyright}

Other than for strictly personal use, it is not permitted to download, forward or distribute the text or part of it, without the consent of the author(s) and/or copyright holder(s), unless the work is under an open content license such as Creative Commons.

\section{Takedown policy}

Please contact us and provide details if you believe this document breaches copyrights.

We will remove access to the work immediately and investigate your claim. 


\title{
WAFER-SCALE GRAPHENE-BASED SOFT ELECTRODE ARRAY WITH OPTOGENETIC COMPATIBILITY
}

\author{
Andrada I. Velea, ${ }^{1,2}$, Sten Vollebregt ${ }^{2}$, Gandhika K. Wardhana ${ }^{1,2}$ and Vasiliki Giagka ${ }^{1,3}$ \\ ${ }^{1}$ Bioelectronics Section, Department of Microelectronics, TU Delft, Delft, THE NETHERLANDS, \\ ${ }^{2}$ Electronic Components, Technology and Materials Section, Department of Microelectronics, TU \\ Delft, Delft, THE NETHERLANDS and \\ ${ }^{3}$ Technologies for Bioelectronics Group, Department of System Integration and Interconnection \\ Technologies, Fraunhofer IZM, Berlin, GERMANY
}

\begin{abstract}
This paper reports on the characterization of a microfabricated wafer-scale, graphene-based, soft implant for spinal cord applications. Graphene is used because of its high transparency and good conductivity, making it suitable for optogenetic applications. Moreover it has a high mechanical strength and is potentially biocompatible. The implant consists of multi-layered chemical vapor deposited graphene, in the form of electrodes and tracks, encapsulated between 2 layers of silicone. Methods such as Raman spectroscopy, optical transmittance, and electrical measurements combined with bending tests and in-vitro experiments, using phosphate-buffered saline (PBS) solution, were employed to characterize the device. The results have shown high bendability and no critical damage of the graphene after immersing the device in PBS solution up to 7 days. To the authors' best knowledge, this is the first work that presents a soft and fully scalable optogenetics-compatible graphene-based spinal cord electrode array.
\end{abstract}

\section{KEYWORDS}

Wafer-scale implant, graphene, silicone, optogenetic compatibility.

\section{INTRODUCTION}

Spinal cord stimulation studies are an important research topic, mostly for pain relief but also for restoring locomotion after spinal cord injuries. Apart from clinical trials, more exploratory research is mostly conducted in rodents. However, the availability of suitable neurotechnologies tailored to small animals is limited. For instance, the spinal cord shows significant mechanical mismatch with rigid neural implants. Even flexible implants encapsulated in stiffer polymers such as polyimide can cause significant damage and compression to the spinal cord [1].

Therefore, effort has been put into the design and fabrication of reliable soft spinal cord implants, based on silicone elastomers.

Existing spinal cord microelectrode arrays (MEAs) feature opaque electrodes $[1,2]$ which are used to electrically activate the tissue or record evoked electrical activity. Alternatively, tissue activation by means of light, i.e. optogenetics, is promising to increase the resolution of activation. This is due to the fact that it activates the tissue in a more type-specific manner. Only neurons that are genetically modified to respond to light will be activated, as long as they are in the vicinity of the light source and the intensity of it is enough to trigger action potentials [3].
To quantify the effect of this new technology there is great need to monitor the electrical response of cells at the site of optogenetic stimulation. To this end, MEAs with transparent electrodes are a necessary tool. In such a system, the transparency will guarantee that light, coming from an external source, can pass through the electrode site, activating the target location. Moreover, the conductivity of MEAs will allow for in situ recording of the evoked electrical response. This temporally and spatially concurrent recording during stimulation is not possible with conventional electrodes, as these systems require both the activating and recorded signals to be in the electrical domain. Transparent parylene-based graphene electrode arrays, with metal tracks, have previously been developed to allow for electrophysiology, in-vivo imaging and optogenetics in the brain [4]. However, parylene, with a Young's modulus of $\sim 2 \mathrm{GPa}$ [5] is still very stiff for the spinal cord.

This work proposes a wafer-scale fabrication process for a silicone-sandwiched graphene-based implant. The materials used ensure the required transparency and conductivity but also provide the necessary softness for interacting with the spinal cord. The design of the proposed implants ensures that any metal layers, if present, only appear at areas which are not under mechanical strain as they have been proven to have poor adhesion to graphene, thus causing delamination [6].

\section{MATERIALS AND METHODS}

The proposed solution was implemented by employing a scalable, 2-mask microfabrication process, inspired by the "flex-to-rigid" (F2R) approach [7], together with a transfer-free CVD process for graphene growth [8] as shown in Fig.1.

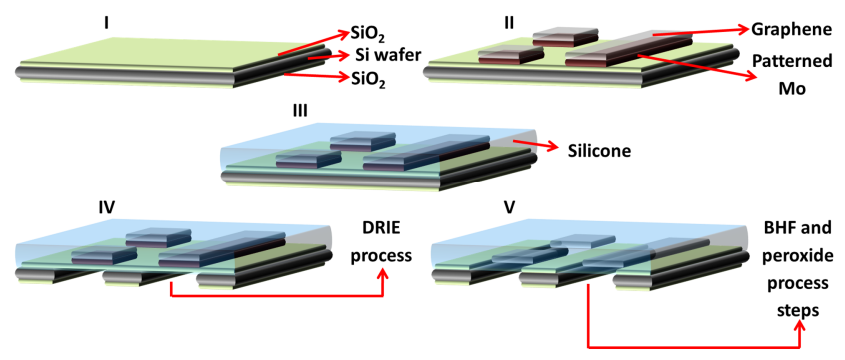

Figure 1: Microfabrication process flow. $\mathrm{SiO}_{2}$ deposition (I). Graphene growth on the molybdenum (Mo) catalyst (II). First layer of silicone spin-coated and cured on top of the structure (III). Release of the complete area using DRIE for Si and wet etching steps to remove the oxide and Mo layers $(I V, V)$. 


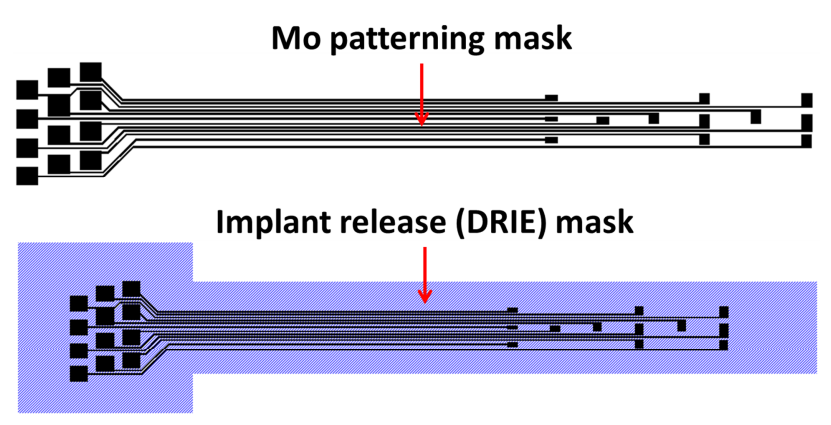

Figure 2: Masks used to develop and suspend the graphene-based passive implants.

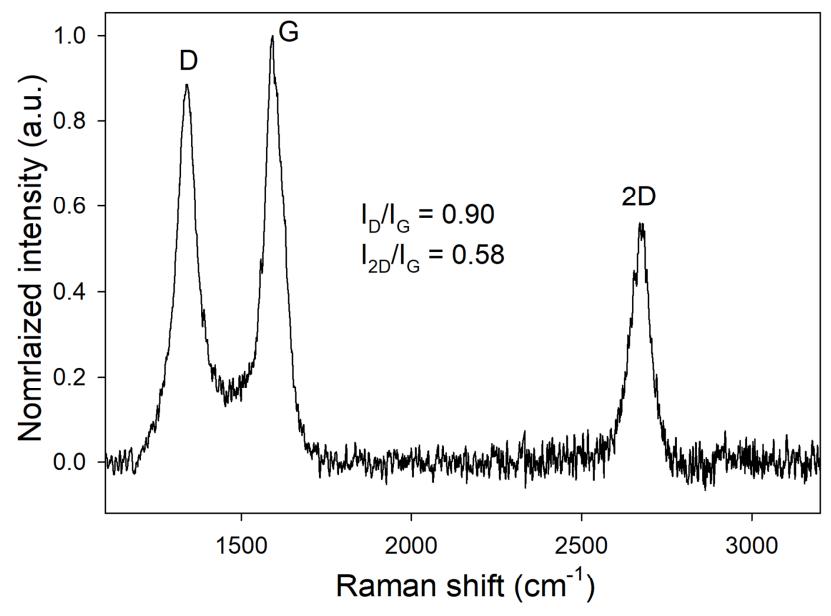

Figure 3: Raman spectroscopy evaluation (using a 633 $n m$ laser) after the $C V D$ process. The $I_{2 D} / I_{G}$ ratio, indicate that a multi-layer graphene has been grown [11].

As the developed prototypes are intended to be used for in-vivo experiments in rats, the MEAs had to be designed accordingly. A total of 12 electrodes were distributed as in Fig. 2, each of them being connected to individual test pads. This number allows for a large coverage of the spinal cord but also fits into the limited space available for implantation in rats [9].

For the microfabrication process, $6 \mu \mathrm{m}$ of plasmaenhanced chemical vapor deposited (PECVD) oxide were deposited on both sides of a double-side polished (DSP) silicon ( $\mathrm{Si}$ ) wafer. Then, on the frontside, $50 \mathrm{~nm}$ of molybdenum (Mo) were sputtered and patterned. This serves as a catalyst for graphene growth. Next, graphene was grown using a CVD process [8] to ensure uniformity of the layer, reproducibility and scalability of the manufacturing.

Before encapsulating the structures in silicone, Raman spectroscopy and two-point measurements were employed to evaluate the graphene layer. Next, $50 \mu \mathrm{m}$ of Sylgard 184 silicone were spin coated on top of the structures and cured for $1 \mathrm{~h}$ at $90{ }^{\circ} \mathrm{C}$. Finally, for the backside of the wafer, a deep reactive ion etching (DRIE) process, was employed only over the areas containing the implants, thus suspending them completely (Fig. 3). This was followed by wet etching of the oxide and Mo layers. Before spin-coating the final encapsulation layer on the backside of the wafer, the structure was again evaluated

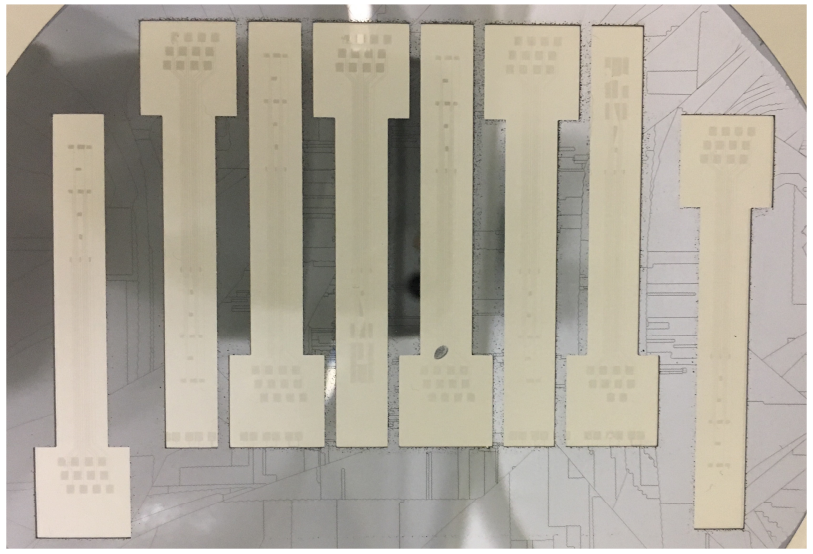

Figure 4: Photograph of the 8 implants fabricated on a single $10 \mathrm{~cm}$ wafer.

by means of Raman spectroscopy and 2-point measurements to ensure that no critical damage was present after the microfabrication process. Moreover, optical transmittance measurements were performed to approximate the number of graphene layers and the transparency of the released graphene-on-silicone structure. In addition, in-vitro experiments were conducted using PBS saline solution for $24 \mathrm{~h}$ and 7 days to quantify how much graphene degrades over time.

The structures were then cut out from the wafers and bending tests were performed by placing the implant around rods with different diameters to emulate handling and deformation expected at the site of implantation. For the actual implant, the final layer of silicone encapsulation can be applied and openings for the electrodes and test pads can be created before removing the samples from the $\mathrm{Si}$ wafer. The robust process achieves high yield and the authors are employing a similar microfabrication process as a basis for the first graphene-based active implants (details can be found in [10]).

\section{RESULTS AND DISCUSSION}

\section{Raman Spectroscopy}

After developing the graphene MEAs on the Si wafer, Raman spectroscopy was employed to determine the presence of graphene and to qualitatively evaluate it. The results in Fig. 3 demonstrate that a multi-layer graphene is present, judging from $\mathrm{I}_{2 \mathrm{D}} / \mathrm{I}_{\mathrm{G}}<1$ [11]. To monitor graphene, Raman spectroscopy was employed after suspending the implants and removing the oxide and Mo layers (the structures are shown in Fig. 4). Also, after conducting the in-vitro experiments, in saline solution, to emulate the biological environment, the graphene layer had to be investigated to evaluate if the graphene is damaged by the PBS solution.

The results in Fig. 5, illustrate that there was no critical damage present on the graphene layer after the microfabrication process. After immersing the structures in PBS, the D peak (indicating the number of defects present) slightly increases. This could be caused by surface contamination of the graphene layer with different particles from the solution. The additional peaks seen on the Raman spectra represent the influence of the silicone Raman signal over the measurement. 


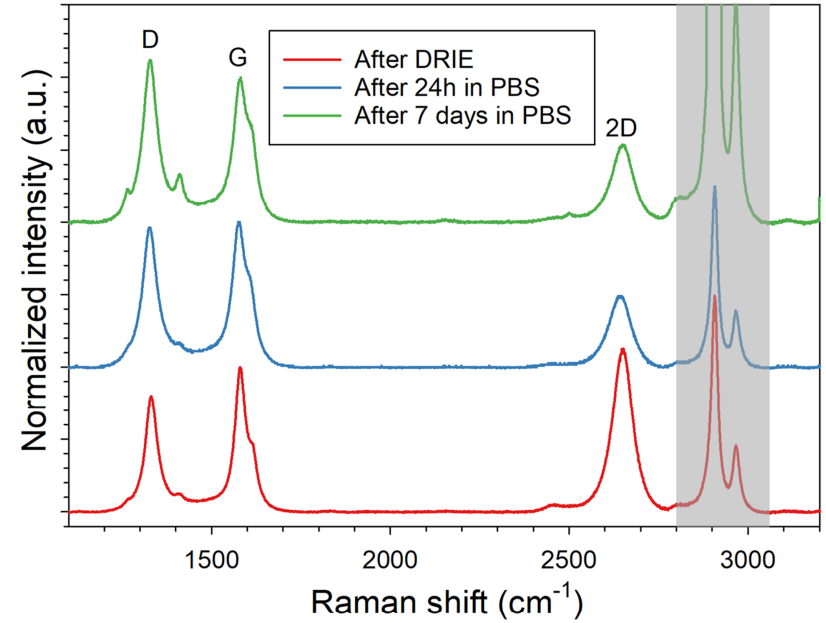

Figure 5: Raman Spectroscopy results DRIE process and after immersing the structure in PBS for $24 h$ and 7 days, respectively. The grey area represents the silicone Raman signal.

\section{Two-Point Measurements}

Apart from Raman spectroscopy, the electrical properties of the graphene were investigated. To this end, two-point measurements were employed after graphene growth (Fig. 6), after suspension of the passive implants (Fig. 6 and Table 1), as well as after conducting the invitro experiments (Table 1). All measurements were performed over a graphene line of $70 \mu \mathrm{m}$ in width and 1 $\mathrm{mm}$ in length.

A significant difference in resistance can be seen from Fig. 6. This originates from the fact that after graphene growth, the measured value is the resistance of both Mo and graphene, whereas for the suspended implants, only the graphene layer was probed as the Mo catalyst had been removed. From this it can be concluded that the resistance of Mo is significantly lower than that of graphene. Moreover, for the suspended implants, the probes were landed directly on graphene which was resting on a $50 \mu \mathrm{m}$ soft silicone substrate, without any metal interface between the measured layer and the twopoint probes. Therefore, the accuracy of the measurement was significantly reduced due to the less controllable contact resistance. Ideally, the graphene track should be measured using a four-point measurement setup to reduce the contact resistance and, on top of that, a metal layer is needed for landing the probes and not damaging the graphene layer. Such designs were not included in the current layout but will be included in future work.

Table 1. Two-point electrical measurement results

\begin{tabular}{|c|c|c|}
\hline Sample type & $\begin{array}{c}\text { Voltage } \\
\text { range }\end{array}$ & $\begin{array}{c}\text { Measured } \\
\text { resistance }\end{array}$ \\
\hline After DRIE & $-5 \mathrm{~V}$ to $5 \mathrm{~V}$ & $200 \mathrm{k} \Omega$ \\
\hline $\begin{array}{c}\text { After DRIE and } \\
24 \mathrm{~h} \text { in PBS }\end{array}$ & $-5 \mathrm{~V}$ to $5 \mathrm{~V}$ & $160 \mathrm{k} \Omega$ \\
\hline $\begin{array}{c}\text { After DRIE and } \\
7 \text { days in PBS }\end{array}$ & $-5 \mathrm{~V}$ to $5 \mathrm{~V}$ & $130 \mathrm{k} \Omega$ \\
\hline
\end{tabular}

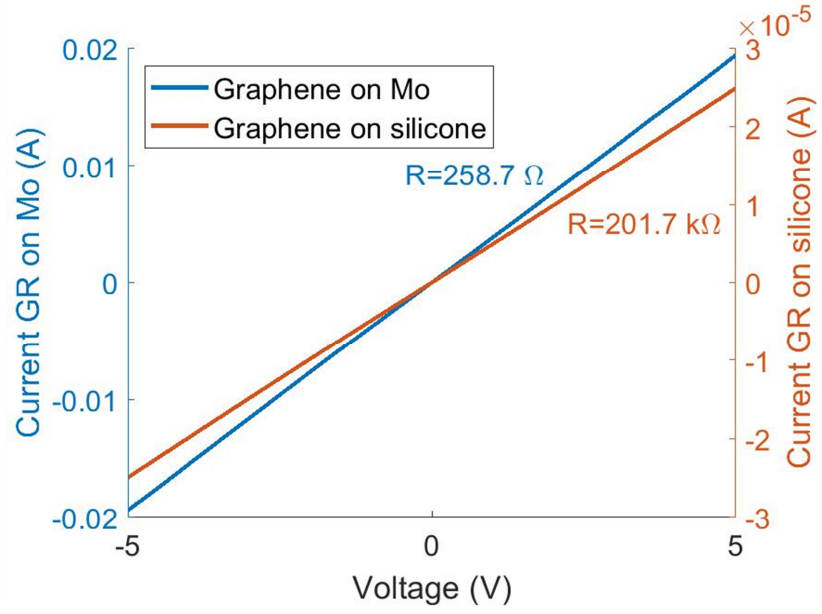

Figure 6: Two-point electrical evaluation of graphene after the CVD process. The results show a resistance value of $\sim 250 \Omega$ for graphene on Mo, and $200 \mathrm{k} \Omega$ for graphene on silicone. The contact resistances from the probes also contribute to the overall resistance.

Having the implants suspended, in-vitro experiments using PBS solution were conducted for periods of $24 \mathrm{~h}$ and 7 days respectively. After each period of time, the samples were electrically evaluated, and the results synthesized in Table 1 demonstrate that graphene does not deteriorate in-vitro. The observed differences likely originate from the inaccuracy of the two-point measurements.

\section{Bending Tests}

As the implantation site for the described structures is subjected to different types and degrees of movements, mechanical evaluation is also needed. To this end, bending tests using metal rods with diameters ranging from $8 \mathrm{~mm}$ down to $3 \mathrm{~mm}$, followed by resistive measurements were employed. In Fig. 7, a passive implant on a $3 \mathrm{~mm}$ metal rod is shown while Table 2 synthesizes the electrical measurements performed after bending the samples on different rods. The measured resistance is $\sim 2 \mathrm{M} \Omega$, which originates from the large length-to-width $(\mathrm{L} / \mathrm{W})$ ratio of the implant and the high contact resistance. Since the probing setup was not tailored for such small prototypes, longer tracks had to be measured such that the probes could properly be landed on the sample under test. However, the results in Table 2, all in the same range, demonstrate that the passive structures can be bent down to $3 \mathrm{~mm}$ without damage.

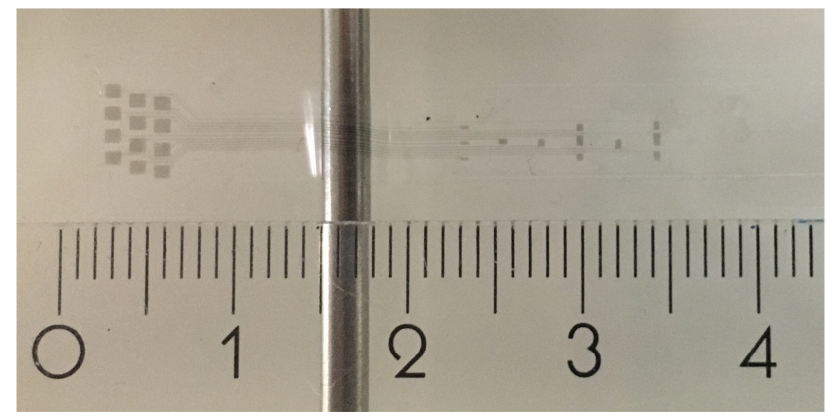

Figure 7: Implant released from the wafer and placed on top of a $3 \mathrm{~mm}$ bending rod. 
Table 2. Bending test results (using two-point electrical measurements)

\begin{tabular}{|c|c|c|}
\hline Sample type & $\begin{array}{c}\text { Voltage } \\
\text { range }\end{array}$ & $\begin{array}{c}\text { Measured } \\
\text { resistance }\end{array}$ \\
\hline No bending & $-5 \mathrm{~V}$ to $5 \mathrm{~V}$ & $1.2 \mathrm{M} \Omega$ \\
\hline Bending $(8 \mathrm{~mm}$ rod) & $-5 \mathrm{~V}$ to $5 \mathrm{~V}$ & $1.8 \mathrm{M} \Omega$ \\
\hline Bending (5 mm rod) & $-5 \mathrm{~V}$ to $5 \mathrm{~V}$ & $2.5 \mathrm{M} \Omega$ \\
\hline Bending (3 mm rod) & $-5 \mathrm{~V}$ to $5 \mathrm{~V}$ & $2.4 \mathrm{M} \Omega$ \\
\hline
\end{tabular}

Note: In case the application requires, this resistance could be lowered (Fig. 5) by leaving the Mo on some parts of the graphene tracks. Using Mo instead of another metal has the advantage that the graphene-Mo bonds are strong, originating from the CVD process.

\section{Optical Transmittance}

As these MEAs are intended to be used in optogenetics, optical transmittance measurements had to be conducted to evaluate the degree of transparency for the final implant. From the optical transmittance result in Fig. 8 it was determined that the implant consists of $\sim 11$ graphene layers and has a transmittance of $72-77 \%$ [12].

\section{CONCLUSIONS}

This paper describes the methods used to evaluate graphene-based passive implants for spinal cord applications. Graphene quality (using Raman), electrical and mechanical characterizations of the final prototypes were performed. Although there were no dedicated structures to evaluate the electrical conductivity of the implants, it has been proven that graphene does not deteriorate and moreover, still conducts after immersing the implants in PBS up to 7 days, as well as after bending them over different rod diameters, down to $3 \mathrm{~mm}$.

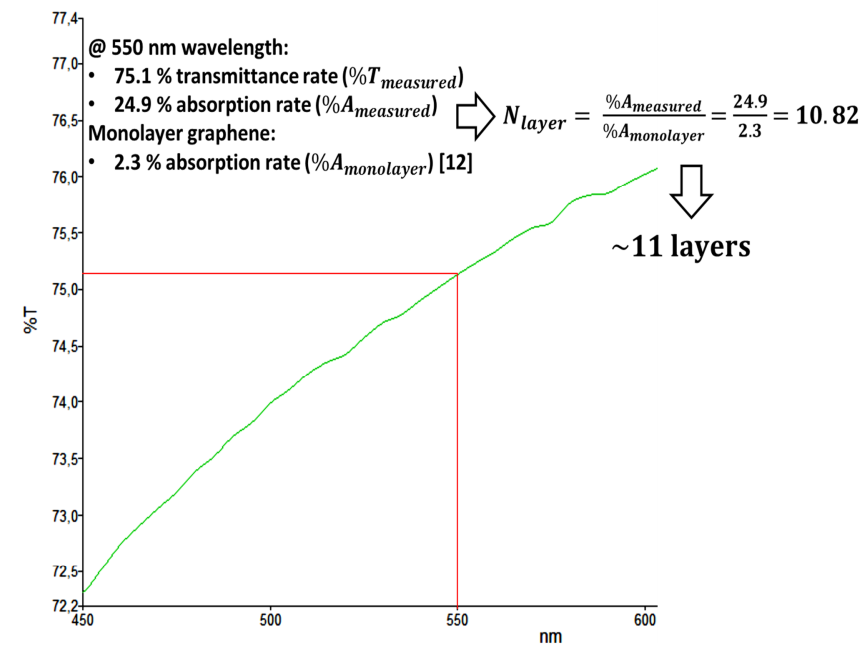

Figure 8: Optical transmittance results for the graphenebased implant. On the $X$-axis, the light wavelength range (visible spectrum). On the Y-axis, the percentage of the light transmitted through the sample.
This paper demonstrates the potential of soft, graphene-based passive implants, to enable unique, currently not available, spinal cord tissue monitoring during optogenetic activation, which is paramount to map the neuronal activation at this level.

\section{ACKNOWLEDGMENTS}

We acknowledge the staff of Else Kooi Laboratory (EKL) and the Bioelectronics group from Delft University of Technology for their support throughout the project.

\section{REFERENCES}

[1] I. R. Minev et al., "Electronic dura mater for longterm multimodal neural interfaces", Science, vol. 347, no. 6218, pp. 159-163, 2015.

[2] V. Giagka, A.Demosthenous and N. Donaldson, "Flexible active electrode arrays with ASICs that fit inside the rat's spinal canal", Biomed. Microdev., vol. 17, no. 6, pp. 106-118, 2015.

[3] K. L. Montgomery et al., "Beyond the brain: Optogenetic control in the spinal cord and peripheral nervous system", Science Transl. Med., vol. 8, no. 337rv5, pp. 1-12, 2016.

[4] D. W. Park et al., "Fabrication and utility of a transparent graphene neural electrode array for electrophysiology, in vivo imaging and optogenetics", Nat. Protoc., vol. 11, no. 11, pp. 2201-2222, 2016.

[5] K. Scholten and E. Meng, "Materials for microfabricated implantable devices: a review", Lab on a Chip, vol. 15, no. 22, pp, 4256-4272, 2015.

[6] J. Robinson et al., "Contacting graphene", Appl. Phys. Lett., vol 98, no. 5, 2011.

[7] B. Mimoun et al., "Flex-to-rigid (F2R): A generic platform for the fabrication and assembly of flexible sensors for minimally invasive instruments", IEEE Sensors, vol. 13, no. 10, pp. 3873-3882, 2013.

[8] S.Vollebregt et al., "A transfer-free wafer-scale CVD graphene fabrication process for MEMS/NEMS sensors", in Proc. IEEE MEMS 2016, Sanghai, China, 2016, pp. 1720.

[9] V. Giagka et al., "Flexible platinum electrode arrays for epidural spinal cord stimulation in paralyzed rats: An in vivo and in vitro evaluation", in Proc. $3^{\text {rd }}$ Annual Conf. IFESSUKI 2012, Birmingham, UK, 2012, pp. 52-53.

[10] A. I. Velea, S. Vollebregt, T. Hosman, A. Pak and V. Giagka "Towards a microfabricated flexible graphenebased active implant for tissue monitoring during optogenetic spinal cord stimulation", in Proc. IEEE NMDC 2019, Stockholm, Sweden, 2019.

[11] Z. Jian et al., "Irradiation effects of graphene and thin layer graphite induced by swift heavy ions", Chinese Phys. B, vol. 24, no. 8, 2015.

[12] K. F. Mak et al., "Measurement of the optical conductivity of graphene", Phys. Rev. Lett, vol. 101, no. 19, pp. 2-5, 2008.

\section{CONTACT}

*A. I. Velea; A.I.Velea@student.tudelft.nl;

*S. Vollebregt; S.Vollebregt@tudelft.nl;

*G. K. Wardhana; G.K.Wardhana@tudelft.nl;

*V. Giagka; V.Giagka@tudelft.nl. 